\title{
Design of surface electromyographic signal acquisition system based on MATLAB
}

\author{
Dan $\operatorname{Ren}^{1, a}$ \\ ${ }^{1}$ College of Engineering \& Technology, Eastern Liaoning University, Dandong Liaoning \\ 118003,China. \\ aldxyrendan@163.com
}

Keywords: sEMG, Design, Data acquisition.

\begin{abstract}
This paper designed the signal acquisition system of surface EMG signal. The hardware part chooses the biological signal acquisition device Thought of Technology company. At the same time, in order to further analysis and the requirement of real-time system, PC communication software based on Matlab. After the experiment, the design of the system is working properly. Provide data analysis for the normal processing of EMG signals, laid a good foundation.
\end{abstract}

\section{Introduction}

Surface electromyography signal(sEMG) is the characterization of skeletal muscle fiber contraction excited, closely related to the impulse of the motor unit biological signals. and muscle activity, has been in the research of computer aided diagnosis with voltage application. sEMG wide value range is generally $0-5 \mathrm{mV}$, muscle contraction the change range of the signal in the $60-300 \mathrm{uV}$ relaxation time will be slightly lower to $20-30 \mathrm{uV}$ within the scope of microvolt sensitivity, so the acquisition equipment is very important for the $\operatorname{system}^{[1-3]}$. At the same time, equipment with good expansibility, easy to develop two times for robot applications, rather than offline signal acquisition.

The design of surface EMG acquisition system including: system hardware equipment selection, selected to satisfy the sampling requirement, has two times the development of good equipment is to build a surface EMG feedback key control loop system rehabilitation robot; acquisition software Matlab environment function and process, based on the clarity of software acquisition system structure clear is the basic conditions for the establishment of the subsequent real-time control.

\section{Acquisition system design}

Equipment acquisition system. The acquisition system equipment is selected in this paper the surface EMG acquisition system as shown in Fig. 1 is composed of ten channel physiological signal acquisition equipment FlexComp Infiniti. Thought Canada technology company developed the equipment to build.

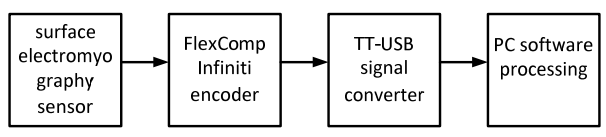

Fig.1 structure of surface EMG signal acquisition system

The surface electromyography sensor is an important part of surface EMG signal acquisition device. MyoScan-Pro surface EMG sensor in this paper, the sensor has three electrodes, anode, cathode and anode and cathode electrode. The shorter distance of the differential input signal collected by the poles, forming a differential signal to resist outside interference the ground electrode; for filtering noise difference signal. The signal through the Myoscan sensor to think is the original signal of surface EMG signal. The input voltage range of the sensor is $0-2000$ VRMS; sensitivity of less than 0.1 VRMS, error is about 0.3 VRMS, the electrode CMRR greater than $100 \mathrm{~dB}$. and the sensor is connected is a one-time surface electrode, the electrode is generally used for medical testing. The electrode material for the silver/silver chloride electrode. The surface is sticky, can better fit on the surface of the skin.

FlexComp Infiniti encoder is equivalent to a high frequency, high resolution data acquisition 
card, which has ten channels. Each channel is relatively independent, in the process of sampling, the channels without mutual interference, noise is relatively small. Each input channel sensor and surface EMG signal Myoscan is connected via signal transmission line special the surface EMG signals collected by sensors. The A/D conversion through the encoder, the incoming optical fiber TT-USB converter. The encoder resolution is $14 \mathrm{bits}$, sampling rate of $2048 \mathrm{~Hz}$. due to the characteristics of surface EMG signal with low frequency, mainly concentrated in the 0 to $500 \mathrm{~Hz}$. According to the calculation formula of the Nyquist frequency, the sampling frequency is far more than the minimum sampling frequency therefore, it can be said that the sampling signal reflects the actual surface EMG signal.

TT-USB signal converter is the function of the optical signal transmitted from the encoder into accord with the requirements of computer USB communication serial communication signal, so that the computer can correctly read and store the surface EMG signals collected by the encoder correctly.

Acquisition system software. The main function of PC software is connected with the encoder, the encoder receives stored data by sending TT-USB sEMG signal converter, real-time rendering of surface EMG. At the same time, the software design adopts the sequence structure is relatively stable, the modules are independent, provide a good environment for the development of the characteristics of real time analysis.

The software design is divided into three parts, ActiveX controls initialization, data acquisition initialization, data acquisition. The structure of the sequence between the parts, is conducive to the software modification, convenient to add a feature extraction algorithm, in order to increase the readability of the program written in various parts of the respective $\mathrm{m}$ file. The main program in the process of running, are calling on them. The overall program flow chart as shown in Fig. 2.

Control initialization. The control part to complete the creation of the ActiveX initialization, the initialization, if an error occurs during the initialization process returns initialization error and close control and hardware connection. In this part, the procedures set up to control some of the basic parameters, such as sensor detection mode, the number of sensors. The flow chart in Fig. 3.

Sample initialization. In the sample before the need to detect sensor channels. These tests include access channel number, channel connection confirmation, channel activation and so on. Similarly, in the setting of error will produce a corresponding warning. The flow chart is shown in Fig. 4.

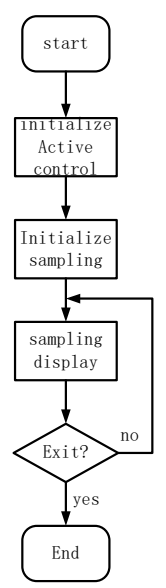

Fig. 2 the general process of the program

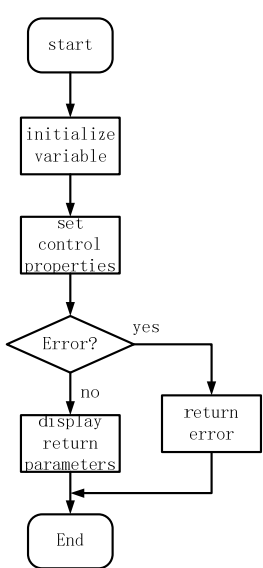

Fig. 3 control initialization flow chart

Data processing. This part is an important part of the software design, realization of data storage and display function of.GUI graphical interface display values on the time axis real return data. Due to the current collection of two channel sEMG signals, time varying signal values are stored in a two-dimensional array of variables called allData. The whole process flow as shown in Fig. 5.

\section{Surface EMG signal acquisition}

Signal acquisition environment. Analysis of surface EMG signals based on actual data, in order to ensure the reliability of the analysis results, combined with the goal of gesture recognition to 
choose the sampling points. Three kinds of static posture and arm movement respectively, elbow flexion, lifting arm, arm and arm exhibition, relaxed posture as target recognition. In order to achieve the recognition function, characteristics of these positions selected surface EMG signals obtained muscle surface measurement, in order to better distinguish motion different, the anterior deltoid, biceps and deltoid, brachioradialis muscle as signal acquisition, feature set up four channel EMG signal. The surface EMG signal acquisition device is connected to the muscle after sampling clean, properly paste electrode surface. Using the PC software to read the surface The return value of the EMG signal.

As a result of the acquisition of the static posture, so start collecting surface EMG signal in the experiment, the subjects need as much as possible to maintain the test requirements of the action. Because the strength intensity will affect the EMG signal, assuming the contra lateral and in the actual training is without load is reasonable in the process of movement in order to avoid giving. Analysis and identification of interference brought in under the condition of static postural muscle strength change. So in the process of acquisition and recognition of the provisions, need to ensure that the subject is not active consciousness of isometric muscle contraction. The sampling frequency is $2048 \mathrm{~Hz}$, each time $20 \mathrm{~s}$.

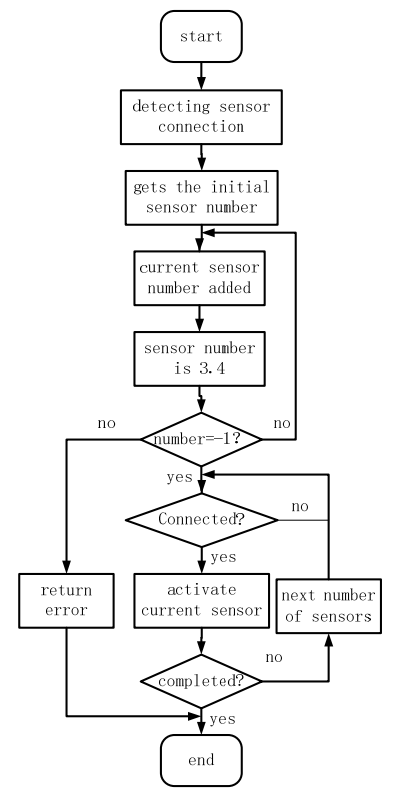

Fig.4 sample initialization

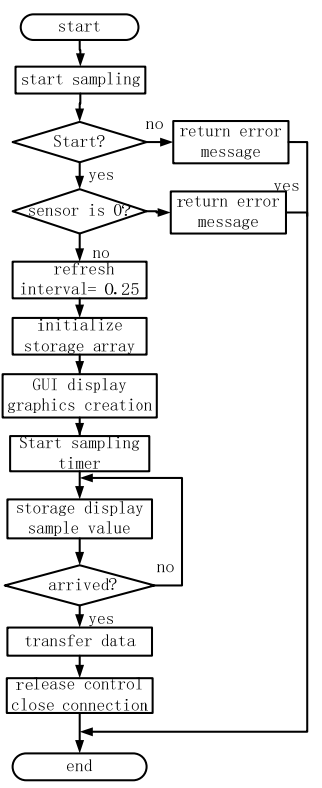

Fig. 5 data process design

Collecting data. In this paper, the design of the host computer software, can be collected to four channels of the original EMG signal data. Fig. 6 shows the elbow flexor movement corresponds to the EMG signal data.

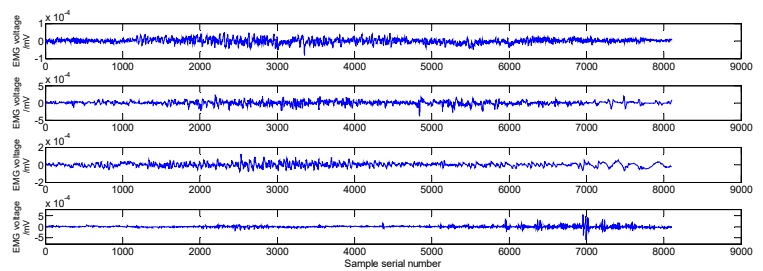

Fig. 6 original data of EMG signal

The data obtained from the collection can be seen:

(1) the amplitude of the EMG voltage is small, the maximum value is $619 \mathrm{uV}$, and the minimum value is $0 \mathrm{uV}$, which indicates that the collected EMG voltage is weak.

(2) the voltage of the EMG signals of the four channels is different, which indicates that the different muscles in the same movement are in different state.

(3) the trend of signal, EMG has variability and random. With different postures, the measured muscle strength change, change range of the EMG signal. At the same time it also verified the surface EMG acquisition system of the building is feasible. 


\section{Conclusions}

This paper designed the signal acquisition system of surface EMG signal. The hardware part chooses the biological signal acquisition device Thought of Technology company. At the same time, in order to further analysis and the requirement of real-time system, PC communication software based on Matlab. After the experiment, the design of the system is working properly. Provide data analysis for the normal processing of EMG signals, laid a good foundation.

\section{References}

[1] Lin Jin-rong, Tan Bei-hai, Xie Sheng-li, Design of ADS1294 SEMG acquisition system. Chinese Medical Equipment Joumal. 36 (2015) 5-7,28.

[2] He Keren, Sun Wei, Luo Jinhong, Zou Ling, Collection and Online Identification System of sEMG. Computer Measurement \& Control.24(2016)241-243,247.

[3] Li Chengkai, Xi Xugang, Wag Junwei, Wu Hao, Acquisition System Design for Surface EMG. Journal of Hangzhou Dianzi University(Natural Sciences) .35(2015) 34-37. 